\title{
1 Is there such a thing as landscape genetics?
}

3 Rodney J. Dyer (RJD)

4

5 Department of Biology \& Center for Environmental Studies

61000 West Cary Street

$7 \quad$ Virginia Commonwealth University

8 Richmond, Virginia 23284-2012

9

10 email: rjdyer@vcu.edu

11 phone: +1 (804) 828-0874

12 fax: +1 (804) 828-0503

13 Running title: Is there such a thing as landscape genetics?

14 Keywords: landscape genetics, landscape ecology, population genetics 


\section{Abstract}

16

17 For a scientific discipline to be interdisciplinary it must satisfy two conditions; it must consist of

18 contributions from at least two existing disciplines and it must be able to provide insights,

19 through this interaction, that neither progenitor discipline could address. In this paper, I

20 examine the complete body of peer-reviewed literature self-identified as landscape genetics

21 using the statistical approaches of text mining and natural language processing. The goal here

22 is to quantify the kinds of questions being addressed in landscape genetic studies, the ways in

23 which questions are evaluated mechanistically, and how they are differentiated from the

24 progenitor disciplines of landscape ecology and population genetics. I then circumscribe the

25 main factions within published landscape genetic papers examining the extent to which

26 emergent questions are being addressed and highlighting a deep bifurcation between existing

27 individual- and population-based approaches. I close by providing some suggestions on where

28 theoretical and analytical work is needed if landscape genetics is to serve as a real bridge

29 connecting evolution and ecology sensu lato. 


\section{Introduction}

32 As the landscape of scientific research and new discovery becomes increasingly

33 interdisciplinary, in both the makeup of diffuse research teams and in the composite nature of

34 hypotheses being examined, it is becoming important to quantify the way in which individual

35 disciplines interact and how new ones contribute to knowledge generation. According to a

36 National Academies report entitled, Facilitating Interdisciplinary Research (2004),

37 interdisciplinary research is "...a mode of research by teams or individuals that integrates

38 information, data, techniques, tools, perspectives, concepts, and/or theories from two or more

39 disciplines or bodies of specialized knowledge..." The purpose of these interactions are to

40 either “...advance fundamental understanding or to solve problems whose solutions are beyond

41 the scope of a single discipline or area of research practice." These specific outcome-orientated

42 restrictions delineate interdisciplinary research from mere collaborations and/or aggregated

43 multidisciplinary work, which may satisfy the composition of the research teams though not fulfill

44 the stated purposes in providing emergent insights. These restrictions beg the question of

45 whether research we are currently conducting and referring to as interdisciplinary is actually

46 addressing emergent questions (heretofore unanswerable using normal approaches from

47 constituent disciplines) or are we simply borrowing approaches and techniques established from

48 different disciplines to answer the same research questions we have been examining for some

49 time.

50

51 As an example, this manuscript looks at a body of research self-identifying as landscape

52 genetics, an emerging keyword designation being applied to research projects studying how

53 ecological, vegetation, anthropogenic, and topographic context influence genetic connectivity 
54 and structure in both natural and modified populations. The moniker was originally introduced

55 by Manel et al. (2003), who provided a definition based upon mechanisms, "The two key steps

56 of landscape genetics are the detection of genetic discontinuities and the correlation of these

57 discontinuities with landscape and environmental features such as barriers." While the

58 mechanics of barrier identification and landscape correlation are not to population genetics

59 (e.g., Dobzhansky 1948; Merriam et al. 1989, Keyghobadi et al. 1999, etc.), the ubiquity of

60 model-based clustering approaches initiated by Pritchard et al. (2000) and the advancements in

61 spatial ecological analysis and GIS technology set the stage for this potentially interdisciplinary

62 research approach. Because of this mix using spatio-ecological data to predict genetic

63 characteristics of organisms (now much broader than just discontinuities), landscape genetics

64 has been characterized as an interdisciplinary fusion of landscape ecology and population

65 genetics (e.g., Manel et al. 2003, Storfer et al. 2006, Holderegger \& Wagner 2008, Balkenhol et

66 al. 2009). Given the focus of these constituent fields and the kinds of research questions being

67 classified as landscape genetics, it is an obvious supposition. However, if landscape genetics

68 is to be correctly defined as an interdisciplinary field, it needs to be more than just the rough

69 interdigitation of progenitor disciplines. Moreover, it must be able to provide insights into

70 processes that are heretofore unattainable-failing to do so would suggest that its continued

71 use would only add an arbitrary and somewhat meaningless categorization to our research

72 descriptions.

73

74 Its flagship journal, Landscape Ecology, defines the discipline itself as an "...interdisciplinary

75 science that focuses explicitly on the ecological understanding of spatial heterogeneity",

76 providing a bit of recursive irony to the definition of landscape genetics. In the preface to the

77 edited volume, Landscape Ecology: A Top-Down Approach, Sanderson \& Harris (2000) suggest

78 the main component that makes landscape ecology distinct from other fields of ecology is that 
79 "...it explicitly encompasses and builds upon the role of heterogeneity in space as well as time."

80 It can be argued that the primary role that landscape ecology has played in the formation of

81 landscape genetics is through analytical advances and integration of sophisticated approaches

82 to quantifying spatial heterogeneity, particularly through the integration of geographic

83 information systems (GIS) into their analyses. In most landscape genetic studies, these

84 techniques provide the basis for the predictor set of variables under consideration. Despite the

85 specifics of the contribution, there is a clear point at which a landscape ecological study will be categorized as landscape genetics; namely when the study includes the use of genetic markers.

Identifying the characteristics of hypotheses that separate landscape genetic studies from population genetic ones is much more difficult. Due in part to its longevity, population genetics has developed a more broad scope of research directions, focusing on both micro- and macro-

91 evolutionary mechanisms. Population genetics, in both theory and practice, examines the

92 mechanisms of evolutionary processes and how they influence within and among groups

93 dynamics. There are clearly kinds of research questions in population genetics that would not

94 be easily mistaken for landscape genetics, as they do not include the environment through

95 which populations are interacting. However, there is a long history of population genetic studies

96 that have specifically included ecological and/or spatial data in the analyses of genetic

97 connectivity and structure (e.g., Smouse et al. 1987, Piertney et al. 1998, Pannell \&

98 Charlesworth 1999) making large fractions of population and landscape genetic studies virtually

99 indistinguishable. As such, the inclusion of spatio-ecological predictor variables alone does not

100 reclassify a population genetic study as landscape genetics and identifying the boundaries

101 separating population from landscape genetics much less clear.

102

103 Applying model-based clustering of genetic information with external landscape and ecological 
104 features may not be sufficient to justify the use of interdisciplinary or to support the de novo

105 creation of a sub-discipline. For that, the outcome of the research must advance fundamental

106 understandings or provide insights that progenitor disciplines could not ascertain. Looking back

107 at the definition, Manel et al. suggest this very thing saying, "Landscape genetics can resolve

108 population substructure across different geographic scales at fine taxonomic levels, thus it is

109 different from the existing understanding of the microevolutionary processes that generate

110 genetic structure across space." At a minimum, this suggests that landscape genetics defines a

111 novel body of knowledge able to identify emergent processes that either landscape ecology or

112 population genetics could not characterize. These processes used in landscape genetics can

113 specifically address the causative ecological and spatial forces influencing the formation and

114 maintenance of genetic structure in a way that was previously unreachable. If true, then

115 landscape genetics is indeed interdisciplinary and it may contain insights that, through

116 introgression of either methodological or theoretical approaches, would benefit both landscape

117 ecology and population genetics greatly. More importantly though, if landscape genetic studies

118 do define a cohesive set of hypotheses and/or approaches that are divergent, then it has the

119 potential to serve as a direct conduit through which fundamental understandings within both

120 Ecology or Evolution (sensu lato) may be exchanged bringing the evolutionary process into

121 tighter connection to the ecological contexts within which they operate. A lofty goal indeed!

123 Here I examine the body of literature defined as landscape genetics to determine if it contains

124 the two components-contributions from two or more disciplines and emergent insights-

125 necessary for it to be considered interdisciplinary research. Rather than summarizing the

126 results from recently published empirical papers (there are already enough review papers on

127 this topic), I instead examine the textual structure of all manuscripts self-identified as landscape

128 genetics using methods of text mining and natural language processing. From these 
manuscripts and a representative sample of ones unambiguously classified as landscape ecology and population genetics, I quantify the composition of the Introduction and Methods sections to determine if the kinds of questions being asked and the ways in which the research

132 is implemented support landscape genetics as an interdisciplinary field addressing emergent

133 hypotheses. In light of the results generated, I discuss the current state of landscape genetics,

134 its relationship to its progenitor disciplines, and provide suggestions for future research

135 directions that aid in understand the interaction of ecological context, spatial heterogeneity, and

136 microevolutionary processes. Moreover, I hope the results presented spark continued

137 development of both theory and applications for this unique intersection of ecological and 138 evolutionary research.

\section{Methods}

\section{Manuscript Collection}

142 Data were collected from literature searches conducted using ISI Web of Science in July 2014.

143 The body of literature designated as landscape genetic (hereafter LG) consisted of the

144 manuscripts that both cited the original Manel et al. (2003) manuscript and specifically self-

145 identified by inclusion of the words "landscape genetic" as a component of the supplied

146 keywords, the title, or contained within the Abstract (n.b., the plural was also used). Similar

147 collections of manuscripts self-identified as landscape ecology (LE) and population genetics

148 (PG) spanning the same time period (2003-2014) were collected. Since the number of LE and

149 PG manuscripts published outnumber LG papers by an order of magnitude, a random sample of

150100 manuscripts were selected from each group. Not all potential manuscripts were available

151 for inclusion, PDF documents from the journals Ecoscience, Israeli Journal of Ecology and 
152 Evolution, Invertebrate Systematics, and the International Journal of Sustainable Development

$153 \&$ World Ecology were behind paywalls and were unavailable to my institution. Manuscripts

154 from Theoretical Population Biology and the American Journal of Physical Anthropology, while

155 available, contained non-conforming PDF encodings making the content unparsable by the

156 software written for this work.

158 Raw text content was preprocessed and prepared for statistical analysis in R (R Core Team, 159 2014) as follows. All PDFs were converted into ascii text format using the footprints library

160 (Dyer, unpublished; available from http://dyerlab.github.com/footprint). Errors in pdf to text conversion (e.g., the loss of a white space separating words, non-ascii ligature translation, etc.) were hand checked and corrected as necessary. Next, the raw text was extracted for both the Introduction and Methods sections separately; to differentiate between the way a manuscript formulates the research question from how the research is implemented. It is implicitly assumed that content in the Results section would be largely redundant to what is presented in the Methods and that the Discussion is where the context of the findings are made, not where key features that differentiating LG from PE and PG are found. All LG papers were classified by hand using four potentially overlapping categories (Table 1): Review, Simulation, Animal, and Plant. Each manuscript could have more than one categorization but no manuscript was left uncharacterized. Only manuscripts determined to be empirical in nature (e.g., those that are not

171 classified as Review) were used in the following analyses. Both LE and PG manuscripts 172 determined as Review were similarly rejected.

174 The textual structure was preprocessed using the $t m$ R package (Feinerer et al. 2008, Feinerer 175 \& Hornick 2014). Raw text was filtered by removing punctuation, numbers, exogenous whitespace, and then converted to lowercase. Common English words (e.g., 'stop-words' as defined 
by Rajaraman \& Ullman 2011) were removed using both the "en" and "SMART" libraries (Lewis 2004). Words were then stemmed using the SnowballC library (Bouchet-Valat 2014) to retrieve their radicals (e.g., the base English word or word component) preventing differences in word tense and alternative suffixes from artificially inflating the error variance term in subsequent

181 discriminant and cluster analysis. Stemmed text content was translated into a multivariate term

182 frequency vector, $\overrightarrow{x_{l}}$. The $\overrightarrow{x_{l}}$ vectors representing the frequency array of stemmed words were 183 then standardized to unit length to minimize bias due to differences in document length as 184 suggested by Dhillon \& Modha (2001) for vector space models. Combined across all documents, the raw data are represented as:

$$
X_{\text {intro }}^{T}=\left[X_{L E}\left|X_{L G}\right| X_{P G}\right]
$$

and

$$
X_{\text {methods }}^{T}=\left[X_{L E}\left|X_{L G}\right| X_{P G}\right]
$$

providing a standardized data matrix for each component of the manuscript representing the frequency distribution of word roots.

\section{Fusion Analysis}

195 To determine the extent to which LG manuscripts are indeed a fusion of LE and PG, a

196 discriminant analysis was performed following Johnson \& Wichern (1992) to derive a set of

197 discriminant functions that maximally separates $X_{L E}$ and $X_{P G}$ Introduction and Methods sections.

198 The discriminant functions were defined on separate covariance estimates for $X_{L E}$ and $X_{P G}$,

199 denoted $\Sigma_{L E}$ and $\Sigma_{P G}$. Then $X_{L G}$ manuscripts were classified, assuming priors equal to the 200 relative frequency of LE and PG papers (denoted as $p_{L E}$ and $p_{P G}$ ) as belonging to the LE 
category when

202

203

and to the PG category when

$$
-\frac{1}{2} x^{\prime}\left(\Sigma_{L E}^{-1}-\Sigma_{P G}^{-1}\right) x+\left(\mu_{L E}^{\prime} \Sigma_{L E}^{-1}-\mu_{P G}^{\prime} \Sigma_{P G}^{-1}\right) x-k \geq \ln \left(\frac{p_{P G}}{p_{L E}}\right)
$$

The applicability of derived discriminant functions were evaluated using the assignment error

210 rate (e.g., the mis-assignment of LE or PG manuscripts). Equality of assignment of LG papers

211 to LE and PG discriminant regions indicating an equal contribution of both progenitor discipline

212 to the body of LG manuscripts, was quantified using a binomial test.

\section{Circumscribing Landscape Genetics}

214 Compositional makeup alone is insufficient to categorize landscape genetics as an

215 interdisciplinary field, it also requires the ability to gain insights that are beyond the scope of the

216 original disciplines. From its inception though, it has been suggested that landscape genetics

217 “...is different from the existing understanding of the microevolutionary processes that generate

218 genetic structure across space." (Manel et al. 2003), satisfying via assertion this requirement.

219 To identify potentially emergent insights that the field of landscape genetics brings, the

220 frequency distribution of stemmed terms in the Introduction and Methods sections of LG, LE,

221 and PG manuscripts were compared to identify terminologies overrepresented in each

222 discipline. Overrepresentation of specific terms may provide inferences into how landscape

223 genetics provides insights into these microevolutionary processes unappreciated in the other 
224 disciplines. Next, the set of self-defined LG manuscripts was examined to determine if they are 225 a single cohesive body or if there are subdivisions within. The vectors in $X_{L G}$ were translated 226 into pairwise Euclidean distance and a hierarchical clustering was performed using Ward's 227 minimum variance method (R Core team, 2014). Confidence on partitions within the clusters 228 was estimated using a bootstrapping approach (via the pvclust R library, Suzuki \& 229 Shimodaira 2011 with 10,000 bootstraps). Differences between major clusters within $X_{L G}$ were 230 then examined qualitatively by estimation of a term frequency matrix and comparison of the 231 most commonly used terms in the main clusters.

\section{Results}

\section{Manuscript Collection}

235 The number of manuscripts citing Manel et al. (2003) listed in ISI Web of Science (WOS) in July 2362014 was 759 . From this group, 288 manuscripts were retained that self-identified by using the 237 term 'landscape genetic(s)' in the title, abstract, or keywords. A similar search on WOS 238 revealed 3,768 PG manuscripts refined using the term "evolutionary biology." From these 239 manuscripts 179 were removed as they contained the term "landscape genetic" or "landscape 240 ecology" in the title, abstract, or keywords and thus would not be appropriate for inclusion in

241 discriminant functions describing putatively pure PG papers. Searches for LE manuscripts

242 refined by "ecology" yielded 3,646 manuscripts, of which 259 also contained the search terms

243 "landscape genetics", "population genetics", or "genetics" in the title, abstract, or keywords and

244 were similarly removed. A random selection of 100 manuscripts defined as non-review were

245 randomly selected from the LE and PG repositories and used for subsequent analyses. All self246 identified LG manuscripts were manually categorized following the definitions in Table 1. By far, 
247 the vast majority of LG studies focus on animal systems $\left(N_{\text {Animal }}=194\right)$ with allocation of

248 manuscripts into all of the remaining categories being roughly equal $\left(N_{\text {Plant }}=61, N_{\text {Review }}=53\right.$,

249 and $\left.N_{\text {Simulation }}=52\right)$. Nearly a quarter $(24 \%)$ of the LG manuscripts were assigned more than

250 one category (Figure 1).

\section{Fusion Analysis}

252 The discriminant functions were relatively well behaved with a total probability of

253 misclassification (e.g., the observed probability of classifying an LE manuscript as PG or vice 254 versa) of $T P M_{\text {Intro }}=0.025$ for the Introduction sections. The classification of LG papers based 255 upon the Introduction were unequal with a relative frequency of classification to PG over LE of $2562: 1\left(p_{P G}=0.65\right.$ vs. $\left.p_{L E}=1-p_{P G}=0.35\right)$, a ratio that deviates significantly from even allocation to 257 both progenitor disciplines (binomial test, $x=91, n=139, P=3.33 e^{-4}, C l_{95}=0.57-0.73$ ). The 258 analysis of LG Methods sections showed both a lower error rate for classification $\left(T P M_{\text {Methods }}=\right.$ 2590.020 ) and a more pronounced bias, a 3:1 ratio, to classify LG papers as PG $\left(p_{P G}=0.81\right)$ instead 260 of $\operatorname{LE}\left(p_{L E}=0.19\right.$; binomial test for equal allocation, $\left.\mathrm{x}=112, \mathrm{n}=139, \mathrm{P}=1.68 \mathrm{e}^{-13}, \mathrm{Cl}_{95}=0.73-0.87\right)$.

261 As a consequence, research manuscripts identified as landscape genetic are much more similar 262 to manuscripts classified as population genetics than to manuscripts identified as landscape 263 ecology. Density estimation for LE, LG, and PG discriminant scores from these analyses shows 264 the extent to which LG is intermediary, though highly skewed, between the distribution defining 265 LE and PG manuscripts (Figure 2).

\section{Circumscribing Landscape Genetics}

267 Differences in term usage between LG, LE, and PG papers (Table 2) highlight the particular 268 focus of landscape genetic studies and how they differ from LE and PG manuscripts. In the 269 Introduction section, the specific word stems distanc, barrier, and connect were all found to be 270 overrepresented in LG manuscripts. That is not to say that these terms were not found in LE or 
271 PG manuscripts, in fact, they occurred at a rate of $0.21,0.09$, and 0.20 for LE papers and 0.16 ,

2720.18 , and 0.15 for PG papers (respectively), they were just more commonly found in LG

273 manuscripts. The Methods sections showed more idiosyncratic differences with only one term

274 found at elevated frequencies in LG manuscripts over both LE and PG: mantel. It is perhaps

275 not surprising given the overrepresentation of distanc, that mantel (the most common way in

276 which distance matrices are evaluated) was found in the Methods section. As in the

277 Introduction section, none of the most overrepresented terms in LG papers were missing from

278 the other manuscripts' categories (LE frequencies range for top LG terms: 0.01-0.41; PG

279 frequency range: 0.03-0.53), they were just used at elevated frequencies.

281 A hierarchical analysis of the Methods section for self-identified LG papers showed clear

282 clustering of manuscripts (Figure 3). Bootstrapping the stemmed terms revealed a total 13

283 separate clusters of manuscripts whose nodes were supported with at least $95 \%$ confidence.

284 The main bifurcation in Figure 3 is just shy of that at $94 \%$ bootstrap support and is indicated as

285 shown. It appears that as a group, there is not a single kind of manuscript that characterizes

286 landscape genetics but rather there are divergent categories. To aid in summarizing the

287 differences between the main groups, the relative frequency of stemmed term usage was

288 estimated to identify overrepresentation of particular terms unique to that group and is depicted

289 by wordcloud inserts for the top ten most overrepresented terms in each cluster. The smallest

290 cluster has only three manuscripts and the relative frequency of term usage should be

291 interpreted with extreme caution, the others have 84 (lower clade) and 52 (upper clade)

292 manuscripts providing a more robust estimation of relative frequency bias. The intersection of

293 term usage in the two large clades reveal the stemmed terms test, distanc, calcul, estim, and

294 data in common. More interesting are the terms not shared among the top ten terms. The

295 terms in the upper clade (in decreasing order of usage) include individu, model, valu, base, and 
compar, whereas the unique terms for the lower clade are sampl, popul, number, studi, and

analysis. The differences seen do not represent words unique to the clade, rather they occur at

an increased frequency when compared to the remaining manuscripts. Of note, and particularly

critical to the differences as depicted by term usage, is that the upper clade emphasis on individu and the lower one that utilizes the term popul, emphasizing potentially divergent foci for research questions. The remaining terms overrepresented in each clade were much more generic including model, valu, base, compar, sampl, number, studi, and analysis.

\section{Discussion}

As a whole, the data show that self-identified landscape genetic papers overwhelmingly resemble studies in population genetics, both in how the questions are formulated (a 2:1 allocation bias) and the methodologies that are used to answer them (a 3:1 bias). The relative term frequency usage for both the Introduction and Methods sections (Table 2) suggest that as a whole, both landscape ecology and population genetics are much broader fields addressing a wider array of question as indicated by the the maximum frequency bias for both landscape ecology and population genetic studies being lower than the top ten terms defining landscape genetics. The differences between landscape ecology and landscape genetics are denoted by

314 the use of terms such as genet, gene, genotyp, dna, linkag, and pcr, clearly related to the 315 addition of genotypic data and highlighting the ease at which the two fields may be 316 differentiated. That is not to say that the history of landscape ecology is entirely devoid of the 317 use of population genetic theory (e.g., Manicacci et al. 1991), the distinction simply provides an

318 identifiable delineation. When compared to population genetics, terms such as landscap, spatial, barrier, and distanc are overrepresented in landscape genetic studies. As previously 
320 noted, it would be a fragile argument to suggest that population genetic studies are distinct from

321 landscape genetic ones because they do not focus on spatial structure, barriers, or the use of

322 distance measures, these were all present in population genetics well before this new moniker

323 was established. Overall, both the discriminant analysis and term usage suggest that to a large

324 degree landscape genetic studies resemble the kinds of questions and approaches commonly

325 called population genetic prior to 2003. These data do suggest that there should be a

326 discussion about the appropriateness of referring to landscape genetics as a unique endeavour

$327 \quad$ versus a subdiscipline residing within population genetics.

An additionally interesting set of bifurcations is found within the totality of landscape genetic

330 studies themselves. As a group, landscape genetics is dominated by animal systems,

331 outnumbering research projects focusing on plant systems or theory \& simulation as well as

332 general review articles (of which there are indeed many) combined (Figure 1). It is at least in

333 part due to the overrepresentation of animal-focused studies that the methodologies used for

334 landscape genetics shows deep bifurcation (Figure 3), particularly as they apply to the

335 distinction between the clade identified by the overrepresentation of individu and the one using

336 popul more frequently. It has been argued that logistical issues associated with life history traits

337 of some kinds of species such as bears (Cushman et al. 2006), moose (Finnegan et al. 2012),

338 pika (Castillo et al. 2014), and tortoise (Hagerty et al. 2011) prevent population-level sampling

339 schemes. It is not to say that certain species cannot be examined at the population level, rather

340 at a more fundamental level, the kinds of questions being asked in these systems are different

341 than those being applied to organisms that are examined within a population level context.

343 Individual-based studies often use genetic markers as a tool to understand transient individual

344 movement across heterogeneous landscapes, particularly focusing on how it is mitigated 
345 through individual behavioral decisions. For example, in the course of introducing the utility of a

346 Causal Modeling approach, Cushman et al. (2006) showed that movement costs based upon

347 inter-individual genetic distances in black bears were most highly correlated with landscape

348 gradients in land cover along specific elevations. They conclude that gene flow, as estimated

349 by individual movement patterns across the sampled landscape, is most common through

350 regions at elevations of $\sim 1,000 \mathrm{~m}$ and through stands of continuous forest canopy. While these

351 findings provide concrete data on demographic movement and aid in the development of

352 conservation and management practices, the authors correctly warn that there is not a direct

353 link between these individual behavioral decisions and actual gene flow, as it is characterized as

354 a microevolutionary process and as population genetic theory defines it. More recently, a study

355 in caribou by Yannic et al. (2013) showed that the strength of the correlation between inter-

356 individual relatedness and ecological separation, a study of isolation by ecological distance,

357 varied dramatically across both year and the sex of the individual being followed. These

358 correlations peaked during calving and rutting periods (maximum Mantel $r=-0.25$ ) and dropped

359 throughout the rest of the year. Even if there are correlations between relatedness and

360 ecological separation fluctuating through time, there does not yet appear to be a theoretical

361 foundation through which quantitative measures gained from individual-based landscape

362 genetic analyses are connected to parameters relevant to evolutionary structure and stability

363 such as $\mathrm{F}_{\mathrm{ST}}$ and $\mathrm{N}_{\mathrm{e}}$.

365 Conversely, approaches that use population-based sampling in landscape genetics do provide a 366 more direct linkage between heterogeneity in the landscape and population genetic structure.

367 This connection is made possible because the bulk of existing microevolutionary theory, what 368 we call population genetics dating from Cotterman (1940), Wright (1943) and Malécot (1948), is 369 based upon population-level inferences. As a result, the parameters estimated to describe 
370 inter-population differentiation, either genetic distance or structure based, have a more direct

371 connection to existing evolutionary theory. While most of the individual based studies are in

372 animals, population-level studies are being applied across all kinds of life histories and sampling

373 approaches. Examples include organisms such as pond-dwelling amphibians (e.g., Murphy et

374 al. 2010, Moore et al. 2011), or among plant groups such as herbs and succulents (e.g., Dyer et

375 al. 2010, Matter et al. 2013), understory trees (e.g., Dyer et al. 2012, DiLeo et al. 2014), or

376 canopy trees (e.g., Diniz-Filho et al. 2009, Poelchau \& Hamrick 2012, Wei et al. 2013).

378 A direct connection between individual processes is largely missing from metrics based upon 379 inter-individual measurements (though see McRae 2006 who argues that ecological separation 380 approximates a coalescent process). Perhaps in response to this, a large portion of empirical 381 landscape genetic studies rely upon stochastic simulation approaches (e.g., Figure 1) as a way 382 to circumvent, either consciously or otherwise, the lack of theoretical connections. The benefit 383 of a simulation-based approach is that one can examine highly specialized situations, though 384 this benefit is paid for by a lack of generality. Simulation alone cannot take the place of theory, 385 a criticism that has been leveled in population genetics as well; running $m s$ is not the same as 386 deriving closed form solutions to population genetic problems. In time, perhaps individual387 based approaches can be reinforced by the development of population genetic theory providing 388 a stronger connection between how transient individual movement translates into quantifiable 389 measurements on processes influencing population genetic structure. However at present, 390 despite the suggestions by Manel et al. (2003), there does not appear to be a robust theoretical 391 basis for how landscape genetics is different from existing bodies of knowledge.

393 Despite the differences between individual- and population-based approaches to landscape 394 genetics, the overlap in the methodologies highlight potentially the main contribution of 
landscape genetics to date, namely the use of distance as a primary analysis tool. Landscape

genetic papers use the stemmed term distanc much more frequently in setting up research

questions ( $+46 \%$ over landscape ecology and $+51 \%$ over population genetics; Table 2$)$. A

similar level of overrepresentation was found in the Methods sections when compared to the sample of population genetic manuscripts $(+43 \%)$.

The key distinction here is that the increased use of distance approaches as a paradigm for analyses has grown tremendously. Distance matrices are used for both predictor (ecological, spatial, topographic) and response (observed genetic data) variables. Mechanistically, there is a wide array of approaches available to quantify the predictor variables, often encoded as spatially explicit raster data, including least-cost paths (e.g., Walker \& Craighead 1997, Graham 2001, Adriaensen 2003), corridor connections (Epps et al. 2007), and all paths (e.g., circuit

407 theory, McRae 2006), to estimate an among-site (or individual as the case may be) distance matrix. As most things in Biology, in certain situations one of these approaches can provide a better fit to the observed genetic data than the others, though all too often alternatives are not tested and one is used based upon supposed 'expert opinion.' Depending upon the kind of

411 raster representation, a suite of new terms such as isolation by landscape resistance (denoted

412 IBR; McRae 2006), isolation by habitat (IBH; Mallet et al. 2014), isolation by ecology (IBE; Wang 413 et al. 2012, Shafer \& Wolf 2013), and isolation by barriers (IBB; Cushman et al. 2006) has

414 sprung into existence. Despite the perceived importance that alternative raster data has, the 415 realized differences in these IB $^{*}$ models are minor compared to the similarity in how we use 416 them. These are essentially models based upon the classic isolation by distance (IBD; Wright

417 1943) paradigm, which had previously been applied to genetic data using spatial (Euclidean)

418 separation. Independent of the differential connection to theory between individual and

419 population level approaches, it is not entirely clear if the proliferation of IB* models is warranted 
420 for more than keyword inflation as they all answer the same general research question. In

421 reality the forces that have produced the observed spatial distribution of genetic structure are

422 most likely due to the interaction of several of these processes overlain through time (e.g., Dyer

423 et al. 2010). It remains to be seen if the application of general IB* frameworks across a broader

424 range of studies results in a reticulation of terminology back to generalized IBD models or

425 progresses towards continued lexicographic fragmentation.

\section{Looking Forward}

427 At a base level, the general model tested in majority of landscape genetic studies can be 428 quantified as:

$$
G \sim f(E)
$$

432 where $G$ is some measure of genetic composition such as pairwise $F_{S T}$ (Rousset 1997; or one

433 of its many analogs), multilocus divergence (Smouse et al. 2001), or one of a variety of potential

434 estimates of genetic frequency (e.g., Weir 1990) or genetic network (Dyer et al. 2010) distance.

435 This response is fit using some model (e.g., the $\sim f()$ part) to the set of predictor variables

436 describing a subset of ecological and/or spatial separation. The variables represented by $E$ can

437 include highly detailed GIS layers, rough approximations of bioclimatic conditions, physical

438 separation based upon distance or barriers, or it can just be populations as a categorical 439 variable.

441 The way in which both $G$ and $E$ are analyzed in landscape genetic studies is most commonly 442 conducted using either correlative approaches such as the single Mantel or Partial Mantel tests 443 (though see Legendre \& Fortin 2010) or via non-parametric regression approaches (Lichstein 
444 2007). The salient distinction of which is that the latter can produce a predicted genetic

445 distance surface (e.g., Dyer et al. 2010, specifically Figure 4 therein) suitable for subsequent

446 hypothesis derivation and testing whereas the former cannot.

448 Comparatively, the level of sophistication that has been developed to create the components of

449 this relationship (i.e., $G, E$, and $\sim f()$ ) is highly skewed. Largely borrowed from landscape

450 ecology, the characterization of physical and ecological separation between observations as

451 depicted in $E$ is highly developed and rather sophisticated. The various IB* predictors and the

452 models within which they are analyzed such as least cost path and circuit theory, are continuing

453 to be developed, are quite novel, and very precise. These developments offer the researcher a

454 wide range of potential predictor variables and variable configurations. To a lesser degree, the

455 statistical approaches based upon the analysis of distance matrices have seen similar

456 development and refinement. Largely, work on understanding how to conduct the analyses has

457 focused on refining the tools we already have; a better Mantel, a more sophisticated Moran's I,

458 etc. The consequence of this is that we've been able to ask the same kinds of questions in a

459 more precise manner, though we are still asking the same questions.

461 Unfortunately, the way in which we characterize $G$ has generally languished. The use of

462 pairwise genetic structure is an aged approach. The use of pairwise structure may be quite

463 robust in the long term but is often too slow to track contemporary processes (e.g., Dyer 2007).

464 Moreover, it often fails to capture the peculiar nature of how contemporary microevolutionary

465 processes such as heterospecific interference in mating (Dyer \& Sork 2001), asymmetry in

466 dispersal (Papa \& Gept 2003), and contemporary consequences of fragmentation (Fore et al.

467 1992) influence genetic variation unless the signal is rather strong (Jaquiéry et al. 2011). Many

468 pairwise genetic distance metrics also come with a set of deep time assumptions (e.g., Roger's, 
469 Nei's, and Bhattacharya's) or have relatively little theory supporting their connection to

470 microevolutionary processes (e.g., Bray-Curtis distance). Other population genetic parameters,

471 less often encountered in landscape genetic studies, such as inbreeding (Diniz-Filho et al. 2009,

472 Guarino \& Cipriani 2013), relatedness or coancestry (Waterhouse et al. 2014), and effective

473 population size (Wang et al. 2011, McCracken et al. 2013) have the potential to provide direct

474 links between landscape processes and established evolutionary theory, though they are often

475 overlooked. One novel approach to characterizing the $G$ side of the equation, distinct from the

476 estimate of pairwise approaches, is the development of network approaches based upon

477 conditional genetic covariance (Dyer \& Nason 2004, Dyer et al. 2010, 2012) where differences

478 are estimated utilizing the entirety of the data set rather than set of pairwise contrasts. While

479 these approaches have allowed the movement beyond classical population genetic parameters,

480 active development in the way in which we characterize population genetic data to bring it to the

481 level at which landscape ecologists have developed spatial data presentation is sorely needed.

482 It is time for the population geneticists to become more involved in landscape genetics. There is

483 a real opportunity here to not only introduce existing tools that are not being utilized to their full

484 potential, but also to push the envelope in how we characterize genetic data and how we

485 connect landscape scale processes (both spatial and temporal) into the existing body of

486 evolutionary theory.

\section{Conclusion}

488 After looking at how landscape genetic studies are portrayed through a textual analysis of 489 published manuscripts, it appears that the field is neither unique nor uniform. It is borrowing 490 disproportionately from population genetics and to a lesser extent landscape ecology. There 491 does not seem to be an indication that landscape genetics addresses questions and hypotheses 492 that other disciplines cannot answer despite the initial suggestion that it does so, relegating it to 
perhaps more multidisciplinary in nature than interdisciplinary.

A reviewer of this manuscript posited that just because the data presented herein does not support the notion that landscape genetics has yielded insights unattainable by either landscape ecology or population genetics, it does not mean that it will not. Indeed, the purpose of this opinion piece is to evaluate the interdisciplinary characteristics of landscape genetics and to look at the body of work in an alternative way with the hopes of finding the components that need additional development. These data shed some light on some of the things that need further development of landscape genetics to live up to what Manel et al. (2003) originally envisioned-undoubtedly more exist.

At a more base level, what we call the kind of science we do may or may not be relevant and this may all be about semantics that at the end of the day are immaterial. My impetus here is to not argue semantics but to understand where additional developments may yield the most reward. As a self-identifying body of research, landscape genetics appears to be currently

508 fragmented. The data presented suggest that there are at least two broad groups roughly 509 partitioned by the focus on individual versus population level processes, both of which seek to 510 address somewhat overlapping sets of hypotheses.

512 Looking forward, there are several potential avenues available to landscape genetics as it

513 continues to mature. First, it could continue on its present course representing a loosely

514 confederated group of different individual and population level studies examining the

515 consequences of heterogeneous landscapes using distance matrices and Mantel tests. This is

516 probably the least beneficial route as it essentially leaves landscape genetics as a grab bag of

517 loosely related questions and approaches. It is also possible that one of the groups may be 
518 subsumed under a yet to be coined moniker, something perhaps all too common in biological

519 sciences, though allowing both sections (individual transient movement vs. effects landscapes

520 have on population genetic structure) to focus more intently on how the specific kinds of

521 hypotheses these approaches provide broad biological relevance. Lastly, and the route for

522 which I advocate, is that the relevant theory connecting individual and population level analyses

523 be developed. While landscape genetics has generally been using population genetic

524 hypotheses and methodologies, it has only been using a small fraction of even the available

525 tools and is ripe ground for continued theoretical development. Independent of which trajectory

526 the field takes, the continued integration of spatial and ecological contexts into the analyses of

527 microevolutionary processes will only aid in tightening the connections between the fields of

528 Ecology and Evolution, sensu lato, and perhaps more importantly spark continued refinement of

529 the kinds of questions we address. 


\section{Acknowledgements}

532

533 This manuscript was adapted from an invited seminar arranged by N. Balkenhol. I also thank A.

534 Eckert, B. Verrelli, and H. Wagner for ongoing discussions on the nature of Landscape Genetics

535 and its relationship to both Landscape Ecology and Population Genetics. 


\section{References}

538

Adriaensen F, Chardon JP, De Blust G, Swinnen E, Villalba S, Gulinck H, Matthysen E. 2003. The application of 'least-cost' modelling as a functional landscape model. Landscape and Urban Planning, 64, 233-247.

Diniz-Filho JAF, Nabout JC, Bini LM, Soares TN, CAmpos Telles MP, Marco Jr P. Collevatti RG. 2009. Niche modelling and landscape genetics of Caryocar brasiliense ("Pequi" tree: Caryocaraceae) in Brazilian Cerrado: an integrative approach for evaluating centralperipheral population patterns. Tree Genetics \& Genomics, 5, 617-627.

Balkenhol N, Gugerli F. Cushman SA, Waits LP, Coulon A, Arntzen JW, Holderegger R, Wagner $\mathrm{HH}$. 2009. Identifying future research needs in landscape genetics: where to from here? Landscape Ecology, 24, 455-463.

Bouchet-Valat M. 2014. SnowballC: Snowball stemmers based on the C libstemmer UTF-8 library. $R$ package version 0.5.1. http://CRAN.R-project.org/package=SnowballC.

Castillo JA, Epps CW, Davis AR, Cushman SA. 2014. Landscape effects on gene flow for a climate-sensitive montane species, the American pika. Molecular Ecology, 23, 843-856.

National Academies. 2004. Facilitating interdisciplinary research. Committee on Facilitating Interdisciplinary Research. 332 pgs. National Academies Press. Washington DC.

Cotterman CW. 1940. A calculus for statistico-genetics. PhD thesis. Ohio State University.

Cushman SA, McKelvey KS, Hayden J, Schwartz MK. 2006. Gene flow in complex landscapes: testing multiple hypotheses with causal modeling. The American Naturalist, 168, 486-499.

Dhillon IS, Modha DS. 2001. Concept decomposition for large sparse text data using clustering. Machine Learning, 42, 143-175.

DiLeo MF, Siu JC, Rhodes MK, López-Villalobos A, Redwine A, Ksiazek K, Dyer RJ. 2014. The gravity of pollination: integrating at-site features into spatial analyses of contemporary pollen movement. Molecular Ecology, 23, 3874-3982.

Dobzhansky T. 1948. Genetics of natural populations. XVI. Altitudinal and seasonal changes produced by natural selection in certain populations of Drosophila pseudoobscura and D. persimilis. Genetics, 33, 158-176.

Dyer RJ, Nason JD. 2004. Population Graphs: the graph theoretic shape of genetic structure. Molecular Ecology, 13, 1713-1727.

Dyer RJ, Nason JD, Garrick RC. 2010. Landscape modeling of gene flow: improved power using conditional genetic distance derived from the topology of population networks. Molecular Ecology, 19, 3746-3759.

Dyer RJ, Chan DM, Gardiakos VA, ws CA. 2012. Pollination graphs: quantifying pollen pool covariance networks and the influence of intervening landscape on genetic connectivity in the North American understory tree, Cornus florida L. Landscape Ecology, 27, 239251.

Epps CW, Wehausen JD, Bleich VC, Torres SG, Brashares JS. 2007. Optimizing dispersal and corridor models using landscape genetics. Journal of Applied Ecology, 44, 714-724.

Feinerer I, Hornik K, Meyer D. 2008. Text Mining Infrastructure in R. Journal of Statistical Software 25(5): 1-54. http://www.jstatsoft.org/v25/i05/.

Feinerer I, Hornik K. 2014. tm: Text Mining Package. R package version 0.6. http://CRAN.Rproject.org/package $=$ tm. 
Finnegan LA, Wilson PJ, Price GN, Lowe SJ, Patterson BR, Fortin MJ, Murray DL. 2012. The complementary role of genetic and ecological data in understanding population structure: a case study using moose (Alces alces). European Journal of Wildlife Research, 58, 415-423.

Graham CH. 2001. Factors influencing movement patterns of keel-billed toucans in a fragmented tropical landscape in southern Mexico. Conservation Biology, 15, 17891798.

Guarino C, Cipriani G. 2013. Landscape discontinuities influence population structure of Acer opalus ssp obtusatum Waldst. \& Kit. ex Willdenow. Plant Biosystems, 147, 1029-1042.

Helfenstein J, Bauer L, Clalüna A, Bolliger J, Kienast F. 2014. Landscape ecology meets landscape science. Landscape Ecology, 29, 1109-1113.

Hennig, C. (2007) Cluster-wise assessment of cluster stability. Computational Statistics and Data Analysis, 52, 258-271.

Hennig, C. (2008) Dissolution point and isolation robustness: robustness criteria for general cluster analysis methods. Journal of Multivariate Analysis, 99, 1154-1176.

Holderegger R, Wagner HH. 2008. Landscape genetics. Bioscience, 58, 199-207.

Hornik K, Feinerer I, Kober M, Buchta C. 2012. Spherical $k$-means clustering. Journal of Statistical Software, 50, 1-22.

Jaquiéry J, Broquet T, Hirzel AH, Yearsley J, Perrin N. 2011. Inferring landscape effects on dispersal from genetic distances: how far can we go? Molecular Ecology, 20, 692-705.

Johnson RA, Wichern DW. 1992. Applied Multivariate Statistical Analysis. Simon \& Shuster, New Jersey.

Keyghobadi N, Roland J, Strobeck C. 1999. Influence of landscape on the population genetic structure of the alpine butterfly Parnassius smintheus (Papilionidae). Molecular Ecology, 8, 1481-1495.

Legendre P, Fortin MJ. 2010. Comparison of the Mantel test and alternative approaches for detecting complex multivariate relationships in the spatial analysis of genetic data. Molecular Ecology Resources, 10, 831-844.

Lewis, DD. 2004. RCV1-v2/LYRL2004: The LYRL2004 Distribution of the RCV1-v2 Text Categorization Test Collection (14-Oct-2005 Version). http://www.jmlr.org/papers/volume5/lewis04a/lyrl2004_rcv1v2_README.htm.

Lichstein JW. 2007. Multiple regression on distance matrices: a multivariate spatial analysis tool. Plant Ecology, 188, 117-131.

Maechler M, Rousseeuw P, Struyf A, Hubert M, Hornik K. 2014. cluster: Cluster Analysis Basics and Extensions. $R$ package version 1.15.2.

Malécot G. 1948. Les Mathématiques de l'Hérédité. Paris. Masson Cie.

Mallet B, Martos F, Blambert L, Pailler T, Humeau L. 2014. Evidence for isolation-by-habitat among populations of an epiphytic orchid species on a small oceanic island. PLoS One, 9, e87469. doi: 10.1371/journal.pone.0087469.

Manicacci D, Olivieri I, Perrot V, Atlan A, Gouyon PH, Prosperi JM, Couvet D. 1991. Landscape Ecology - Population genetics at the metapopulation level. Landscape Ecology, 6, 147-159.

Matter P, Kettle CJ, Ghazoul J, Pluess AR. 2013. Extensive contemporary pollen-mediated gene flow in two herb species, Ranunculus bulbosus and Trifolium montanum, along an altitudinal gradient in a meadow landscape. Annals of Botany, 111, 611-621.

McCracken GR, Perry R, Keefe D, Ruzzante DE. 2013. Hierarchical population structure and genetic diversity of lake trout (Salvelinus namaycush) in a dendric system in Northern Labrador. Freshwater Biology, 58, 1903-1917.

McRae BH. 2006. Isolation by resistance. Evolution, 60, 1551-1561. 
Merriam G, Kozakiewicz M, Tsuchiya E, Hawley K. 1989. Barriers as boundaries for metapopulations and demes of Peromyscus leucopus in farm landscapes. Landscape Ecology, 2, 227-235.

Moore JA, Tallmon DA, Nielsen J, Pyare S. 2011. Effects of landscape on boreal toad gene flow: does the pattern-process relationship hold true across distinct landscapes at the northern range margin? Molecular Ecology, 20, 4858-4869.

Murphy MA, Dezzani R, Pilliod DS, Storfer A. 2010. Landscape genetics of high mountain frog metapopulations. Molecular Ecology, 19, 2624-2649.

Pannell JR, Charlsworth B. 1999. Neutral diversity in a metapopulation with recurrent local extinction and recolonization. Evolution, 53, 664-676.

Paradis E., Claude J. \& Strimmer K. 2004. APE: analyses of phylogenetics and evolution in R language. Bioinformatics, 20, 289-290.

Piertney SB, MacColl ADC, Bacon PJ, Dallas JF. 1998. Local genetic structure in red grouse (Lagopus lagopus scoticus): evidence from microsatellite DNA markers. Molecular Ecology, 7, 1645-1654.

Poelchau MF, Hamrick JL. 2012. Differential effects of landscape-level environmental features on genetic structure in three codistributed trees species in Central America. Molecular Ecology, 21, 4970-4982.

Pritchard JK, Stephens M. Donnelly P. 2000. Inference of population structure using multilocus genotype data. Genetics, 155, 945-959.

R Core Team (2014). R: A language and environment for statistical computing. R Foundation for Statistical Computing, Vienna, Austria. URL http://www.R-project.org/.

Rajaraman, A.; Ullman, J. D. (2011). "Data Mining". Mining of Massive Datasets. pp. 1-17. doi:10.1017/CBO9781139058452.002.

Rousset F. 1997. Genetic differentiation and estimation of gene flow from F-statistics under isolation by distance. Genetics, 145, 1219-1228.

Sawaya MA, Kalinowski ST, Clevenger AP. 2014. Genetic connectivity for two bear species at wildlife crossing structures in Banff National Park. Proceedings of the Royal Society: BBiological Sciences, 281, 20131705.

Sanderson J, Harris LD. 2000. Landscape Ecology: A Top-Down Approach. Lewis Publishers, Washington DC.

Shafer AB, Wolf JBW. 2013. Widespread evidence for incipient ecological speciation: a metaanalysis of isolation-by-ecology. Ecology Letters, 16, 940-950.

Smouse PE, Wood JW. 1987. The genetic demography of the Gainj of Papua New Guinea III: Functional models of migration and their genetic implications. In: Mammalian dispersal patterns: the effects of social structure on population genetics. Chepko-Sade BD, Halpin Z. Eds. University of Chicago Press. Chicago, IL.

Smouse PE, Dyer RJ, Westfall RD, Sork VL. 2001. Two-generation analysis of pollen flow across a landscape I. Male gamete heterogeneity among females. Evolution, 55, 260271.

Storfer A, Murphy MA, Evans JS, Goldberg CS. 2006. Putting the 'landscape' in landscape genetics. Heredity, 98, 128-142.

Suzuki R, Shimodaira H. 2011. pvclust: Hierarchical clustering with p-values via multiscale boostrap resampling. R package version 1.2-2. http://CRAN.rproject.org/package=pvclust.

Walker R, Craighead L. 1997. Corridors in Montana using GIS. In: Proceedings of the ESRI European User Conference on Analyzing Wildlife Movement. Pgs. 1-18. Copenhagen.

Waterhouse MD, Sloss BL, Isermann DA. 2014. Relationship among Walleye population characteristics and genetic diversity in Northern Wisconsin lakes. Transactions of the 
American Fisheries Society, 143, 744-756.

680 Wang IJ, Johnson JR, Johnson BB, Shaffer HB. 2011. Effective population size is strongly correlated with breeding pond size in the endangered California tiger salamander, Ambystoma californiense. Conservation Genetics, 12, 911-920.

Wang IJ, Glor RE, Losos JB. 2012. Quantifying the roles of ecology and geography in spatial genetic divergence. Ecology Letters, 16, 175-182

Weir BS. 1990. Genetic data analysis. Methods for discrete population genetics. 377 pages. Sinauer \& Associates. Sunderland MA.

Wei X, Meng H, Jiang M. 2013. Landscape genetic structure of a streamside tree species, Euptelea pleisopermum (Eupteleaceae): Contrasting roles of river valley and mountain ridge. PLoS One, 8, e66928.

Wright S. 1943. Isolation by distance. Genetics, 28, 114-138.

Yannic G, Pellissier L, Le Corre M, Dussault C, Bernatchez L, Côté SD. 2014. Temporally dynamic habiat suitability predicts genetic relatedness among caribou. Proceedings of the Royal Society: B-Biological Sciences, 281, 20150502. 


\section{Data Accessibility}

695

696 The data and scripts necessary to perform all analyses in this manuscript are posted to github.

697 The $\mathrm{R}$ library footprint is located at https://github.com/dyerlab/footprints and the scripts and data

698 for this paper hosted at https://github.com/dyerlab/ITSATALG. Both components are available

699 under an open source license. 
700 Table 1: Topic categories used to classify all self-identified landscape genetic manuscripts.

701 Each manuscript was ascribed at least one of these categories.

702

\begin{tabular}{|c|c|}
\hline Category & Criteria \\
\hline Review & $\begin{array}{l}\text { The manuscript contained a review of existing landscape genetic studies and/or } \\
\text { how landscape genetics could be leveraged into an existing field of study }\end{array}$ \\
\hline Simulation & $\begin{array}{l}\text { The manuscript either conducted simulations and/or modeling as a component } \\
\text { of the work or introduced novel statistical approach requiring the development } \\
\text { of new approaches }\end{array}$ \\
\hline Animal & The manuscript analyzed an empirical data set from an animal species \\
\hline Plant & The manuscript analyzed a data set from a plant, algal, and/or fungal species. \\
\hline
\end{tabular}

703 
704 Table 2: Differences in the frequency of stemmed terms comparing landscape genetic (LG) to 705 both Landscape Ecology (LE) and Population Genetic (PG) manuscripts in both (A) Introduction, and (B) Methods sections. Numerical value next to stemmed term represents the frequency

708

709 (A) Introduction

\begin{tabular}{|c|c|c|c|c|c|c|c|}
\hline \multicolumn{4}{|c|}{ Landscape Ecology } & \multicolumn{4}{|c|}{ Population Genetics } \\
\hline$+\mathrm{LC}$ & & $+\mathrm{LE}$ & & $+L G$ & & $+P G$ & \\
\hline genet & 0.89 & veget & 0.34 & landscap & 0.94 & evolut & 0.37 \\
\hline gene & 0.88 & resourc & 0.26 & spatial & 0.58 & genom & 0.30 \\
\hline barrier & 0.53 & properti & 0.24 & featur & 0.51 & sequenc & 0.27 \\
\hline dispers & 0.53 & densiti & 0.24 & distanc & 0.51 & morpholog & 0.27 \\
\hline differenti & 0.52 & soil & 0.24 & connect & 0.50 & mutat & 0.23 \\
\hline flow & 0.48 & activ & 0.23 & habitat & 0.50 & histori & 0.21 \\
\hline geograph & 0.47 & abund & 0.22 & structur & 0.48 & trait & 0.21 \\
\hline distanc & 0.46 & communiti & 0.22 & dispers & 0.47 & origin & 0.17 \\
\hline connect & 0.45 & control & 0.21 & barrier & 0.44 & genus & 0.16 \\
\hline isol & 0.44 & matter & 0.20 & movement & 0.41 & fitness & 0.16 \\
\hline
\end{tabular}


711 Table 2 (cont.)

712

713 (B) Methods

Landscape Ecology

\begin{tabular}{|c|c|c|c|}
\hline \multicolumn{2}{|c|}{$+L G$} & \multicolumn{2}{|c|}{$+\mathrm{LE}$} \\
\hline genotyp & 0.83 & day & 0.33 \\
\hline pairwis & 0.81 & veget & 0.32 \\
\hline gene & 0.81 & open & 0.32 \\
\hline dna & 0.79 & survey & 030 \\
\hline mantel & 0.78 & soil & 0.29 \\
\hline differenti & 0.78 & land & 0.29 \\
\hline equilibr & 0.77 & normal & 0.28 \\
\hline linkag & 0.77 & domin & 0.28 \\
\hline pcr & 0.76 & composit & 0.27 \\
\hline popul & 0.73 & annual & 0.26 \\
\hline
\end{tabular}

Population Genetics

\begin{tabular}{|c|c|c|c|}
\hline \multicolumn{2}{|c|}{$+L G$} & \multicolumn{2}{|c|}{$+P G$} \\
\hline landscap & 0.80 & align & 0.34 \\
\hline spatial & 0.62 & substitut & 0.31 \\
\hline mantel & 0.55 & phylogenet & 0.26 \\
\hline area & 0.48 & neutral & 0.19 \\
\hline habitat & 0.48 & evolut & 0.19 \\
\hline dispers & 0.44 & segreg & 0.19 \\
\hline barrier & 0.44 & recombin & 0.18 \\
\hline connect & 0.43 & experi & 0.18 \\
\hline correl & 0.43 & phenotyp & 0.17 \\
\hline distanc & 0.43 & ident & 0.16 \\
\hline
\end{tabular}

714 
bioRxiv preprint doi: https://doi.org/10.1101/018192; this version posted April 16, 2015. The copyright holder for this preprint (which was not certified by peer review) is the author/funder, who has granted bioRxiv a license to display the preprint in perpetuity. It is made available under aCC-BY-ND 4.0 International license.

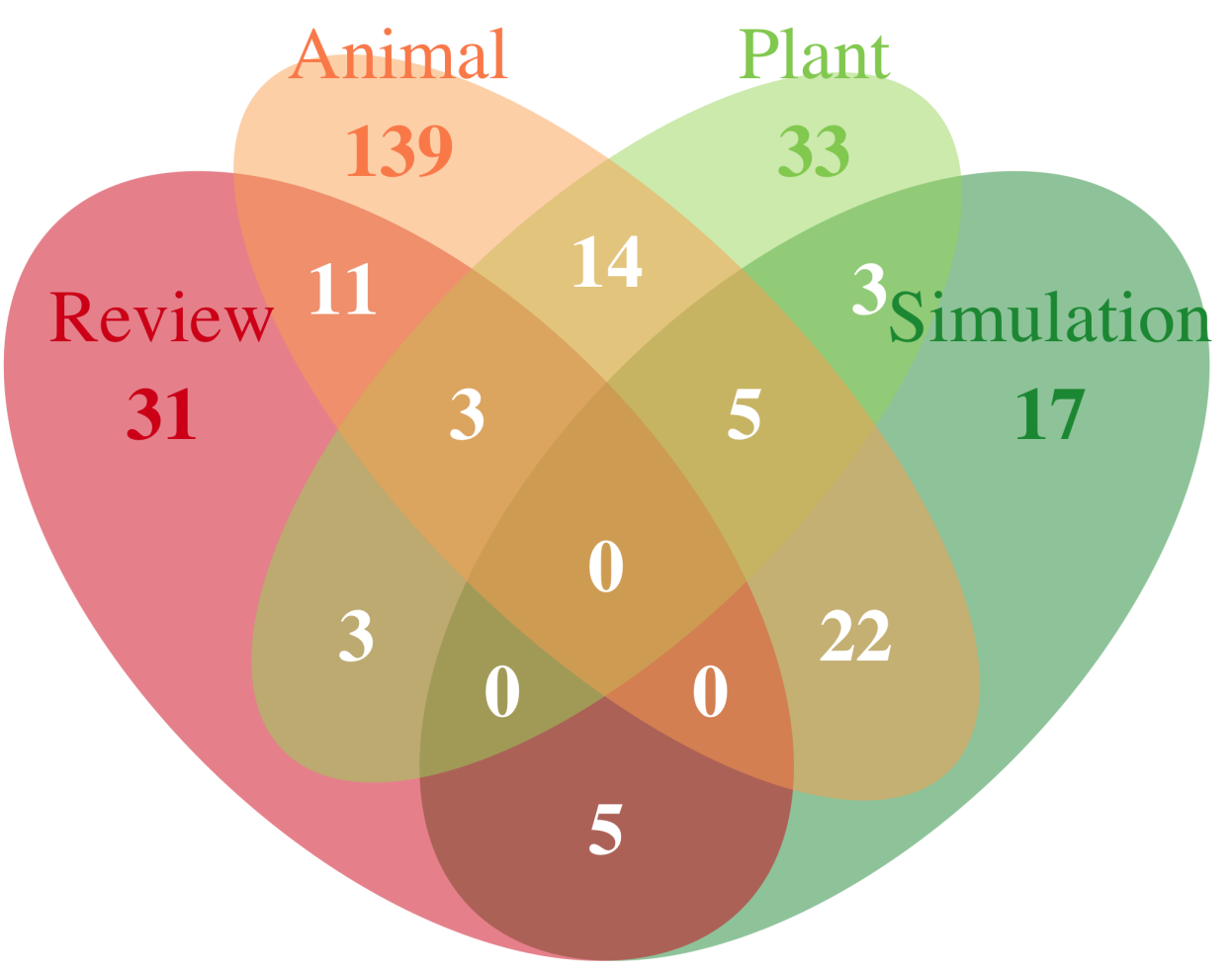

724 Figure 1: Classification of manuscripts self-identified as 'landscape genetics' into Review, 725 Simulation, Animal, and Plant categories (see Table 1 for a complete description). 
bioRxiv preprint doi: https://doi org/10,1101/018192; this version posted April 16, 2015. The copyright holder for this preprint (which was not certified by peer review) is the author/funder, who has granted bioRxiv a license to display the preprint in perpetuity. It is made available under aCC-BY-ND 4.0 International license.

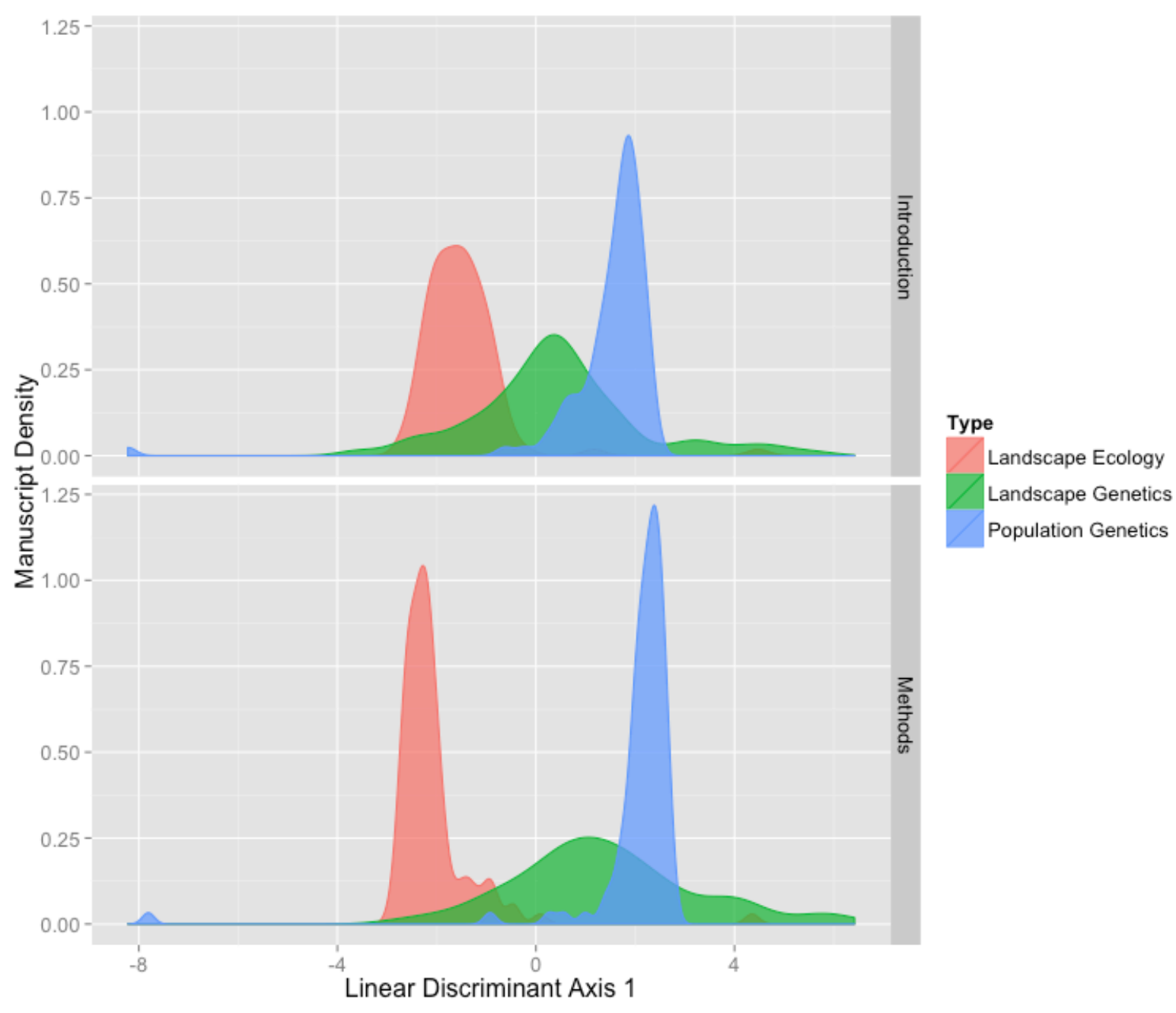

732 Figure 2: Density plot of discriminant scores for LE, LG, and PG papers defined by the contents of the Introduction (top) and Methods (bottom) sections. The majority of LG papers were classified as PG for both the Introduction (65\%) and Methods (78\%) textual components. 


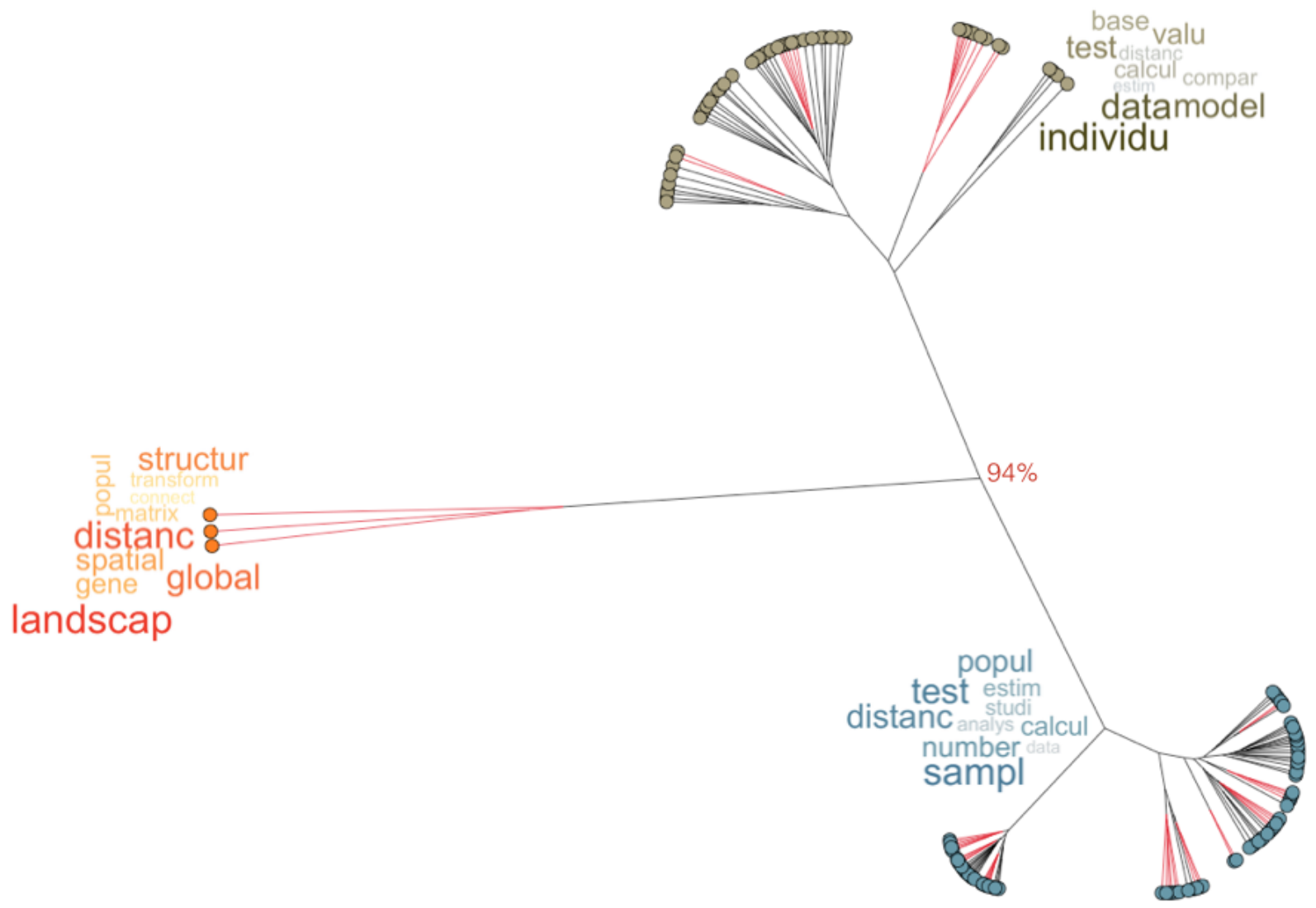

740 Figure 3: Hierarchical clustering using Ward's minimum variance metric of stemmed text content 741 found within the Methods section of self-identified landscape genetic papers. Clusters with $742>95 \%$ bootstrap support are shown with red branches (the main bifurcation separating the three 743 groups had $94 \%$ bootstrap support as indicated). The relative frequency of to the top 10 744 stemmed words are shown as wordcloud inserts (term font size represents decreasing order of 745 usage). 